\title{
The ISW imprints of voids and superclusters on the CMB
}

\author{
S. Hotchkiss ${ }^{1}, \dagger$ S. Nadathur ${ }^{2}$, S. Gottlöber ${ }^{3}$, I. T. Iliev ${ }^{1}$, A. Knebe ${ }^{4}$, \\ W. A. Watson ${ }^{1}$ and G. Yepes ${ }^{5}$ \\ ${ }^{1}$ Department of Physics and Astronomy, University of Sussex, Falmer, Brighton, \\ BN1 9QH, UK \\ ${ }^{2}$ Department of Physics, University of Helsinki and Helsinki Institute of Physics, P.O. Box 64, \\ FIN-00014, University of Helsinki, Finland \\ ${ }^{3}$ Leibniz-Institute for Astrophysics, An der Sternwarte 16, D-14482 Potsdam, Germany \\ ${ }^{4}$ Departamento de Física Teórica, Modulo C-XI, Facultad de Ciencias, Universidad Autónoma \\ de Madrid, 28049 Cantoblanco, Madrid, Spain
}

\begin{abstract}
We examine the stacked integrated Sachs-Wolfe (ISW) imprints on the CMB along the lines of sight of voids and superclusters in galaxy surveys, using the Jubilee ISW simulation and mock luminous red galaxy (LRG) catalogues. We show that the expected signal in the concordance $\Lambda \mathrm{CDM}$ model is much smaller than the primary anisotropies arising at the last scattering surface and therefore any currently claimed detections of such an imprint cannot be caused by the ISW effect in $\Lambda$ CDM. We look for the existence of such a signal in the Planck CMB using a catalogue of voids and superclusters from the Sloan Digital Sky Survey (SDSS), but find a result completely consistent with $\Lambda \mathrm{CDM}$ - i.e., a null detection.
\end{abstract}

Keywords. cosmology: cosmic microwave background, dark energy, large-scale structure of Universe, methods: numerical, methods: data analysis

\section{Introduction}

The possibility of detecting the ISW imprints on the CMB of voids and superclusters using stacking techniques has received much attention following the work of Granett et al. (2008) (hereafter G08), who claimed a very high-significance $(>4 \sigma)$ detection of correlation between superstructure lines-of-sight and corresponding hot and cold spots on the CMB. The magnitude of the claimed signal, $\sim 10 \mu \mathrm{K}$, has however always been puzzlingly large (Hunt \& Sarkar (2010), Granett et al. (2009)). Theoretical estimates (Nadathur et al. (2012)) suggested that the maximum possible stacked ISW signal in a $\Lambda \mathrm{CDM}$ cosmology should be an order of magnitude smaller, a conclusion later supported by estimates based on $N$-body simulations (Flender et al. (2013), Hernández-Monteagudo \& Smith (2013), Cai et al. (2013)).

Various consistency checks of the original detection failed to identify any systematic effects or foregound contamination (Hernández-Monteagudo \& Smith (2013)), suggesting a cosmological origin for the effect, which was also found to persist in Planck CMB data (Planck Collaboration (2013a)). The use of an alternative catalogue of voids alone, drawn from independent SDSS data at lower redshift, did not support the original highsignificance detection (Ilić et al. (2013),Planck Collaboration (2013a)), but this catalogue was later shown to be flawed (Nadathur \& Hotchkiss (2014), hereafter NH14). Another measurement using a better void catalogue (Cai et al. (2013)) also gave inconclusive

\section{$\dagger$ s.a.hotchkiss@sussex.ac.uk}



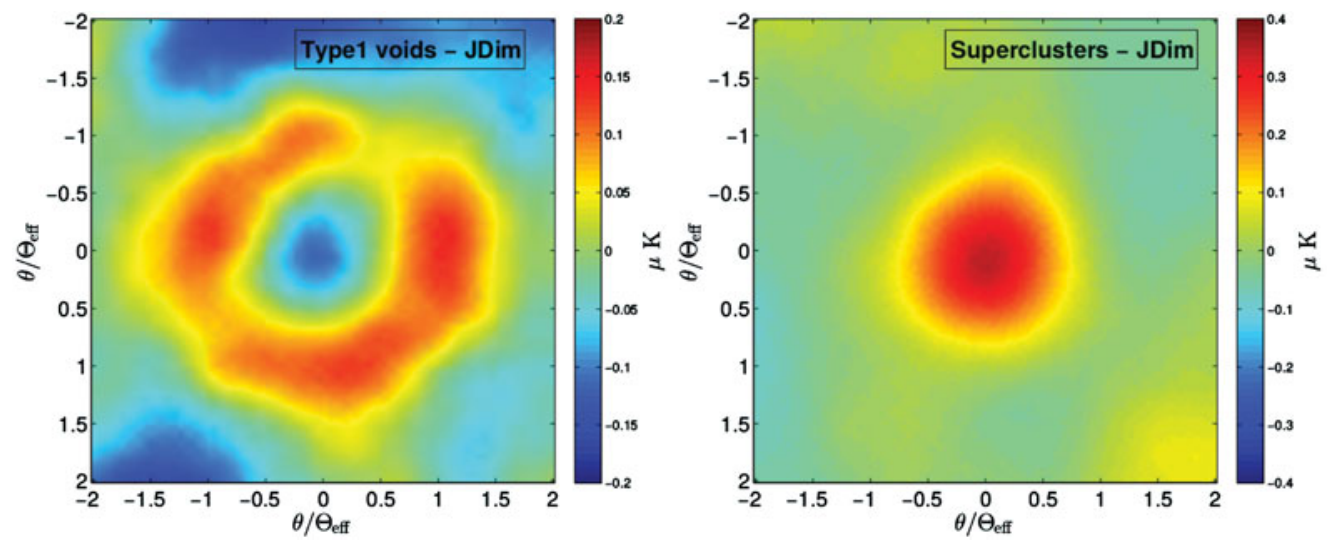

Figure 1. Stacked and rescaled patches from the simulated Jubilee ISW maps along the directions of structures identified in the JDim catalogue. Largest scale modes $(\ell \leqslant 10)$ have been removed for clarity. Left: Type1 voids; right: superclusters.

results, claiming a possible detection of an anomalously large ISW signal from stacked voids, but at low $\mathrm{S} / \mathrm{N}(\sim 2 \sigma$ significance $)$.

We re-examine this issue using the Jubilee ISW simulation as well as the Planck SMICA map (Planck Collaboration (2013b) and a robust catalogue of structures from the SDSS DR7 LRG samples (NH14). Unlike more recent studies but in the spirit of the original claimed detection, we make use of both voids and superclusters in an attempt to maximize any possible $\mathrm{S} / \mathrm{N}$.

\section{Method}

We make use of the Jubilee ISW simulation, which contains $6000^{3}$ particles in a $\left(6 h^{-1} \mathrm{Gpc}\right)^{3}$ box, with a cosmology based on the WMAP5 results (Dunkley et al. (2009)): $\Omega_{m}=0.27, \Omega_{\Lambda}=0.73, \Omega_{b}=0.044, h=0.72, \sigma_{8}=0.8$ and $n_{s}=0.96$. Initial conditions of the simulation are set at redshift $z=100$. Full-sky maps of the ISW temperature anisotropy are constructed (see Watson et al. (2014) for details) for an observer at the centre of the box by ray-tracing photons through the gravitational potential according to $\Delta T(\hat{\mathbf{n}})=2 \bar{T}_{C M B} \int \dot{\Phi}(r, \hat{\mathbf{n}}) a d r$, where $\dot{\Phi}$ is calculated from the potential $\Phi$ using the semi-linear approximation $\dot{\Phi}=-\Phi H(t)[1-\beta(t)]$ (Cai et al. (2010)), where $\beta=d \ln D / d \ln a$ and $D(t)$ is the linear growth function. The large size of the Jubilee simulation means that these maps are complete for all structures out to $z \sim 1.4$ without repetition of the box.

Within the Jubilee simulation, halos are resolved on the light cone down to a mass of $\sim 1.5 \times 10^{12} h^{-1} M_{\odot}$ using a spherical overdensity algorithm. We use the HOD model of Zheng et al. (2009) to populate these halos in order to obtain two mock LRG catalogues JDim and JBright between redshifts $z \in(0.16,0.36),(0.16,0.44)$, as described by Watson et al. (2014), Hotchkiss et al. (2014). These are designed to match the SDSS LRG catalogues of Kazin, et al. (2010). To identify voids and superclusters within these catalogues we use a modification of the ZOBOV algorithm (Neyrinck (2008)). Our methodology closely follows that outlined by NH14, including the use of buffer particles at the survey boundaries. Selection criteria for the final superstructures are chosen to match those used for Type1 and Type2 voids and superclusters introduced in that paper. Our catalogues of SDSS structures are taken from the public catalogues of $\mathrm{NH} 14 \dagger$, who found structures in

$\dagger$ Available for download from www.hip.fi/nadathur/download/dr7catalogue. 

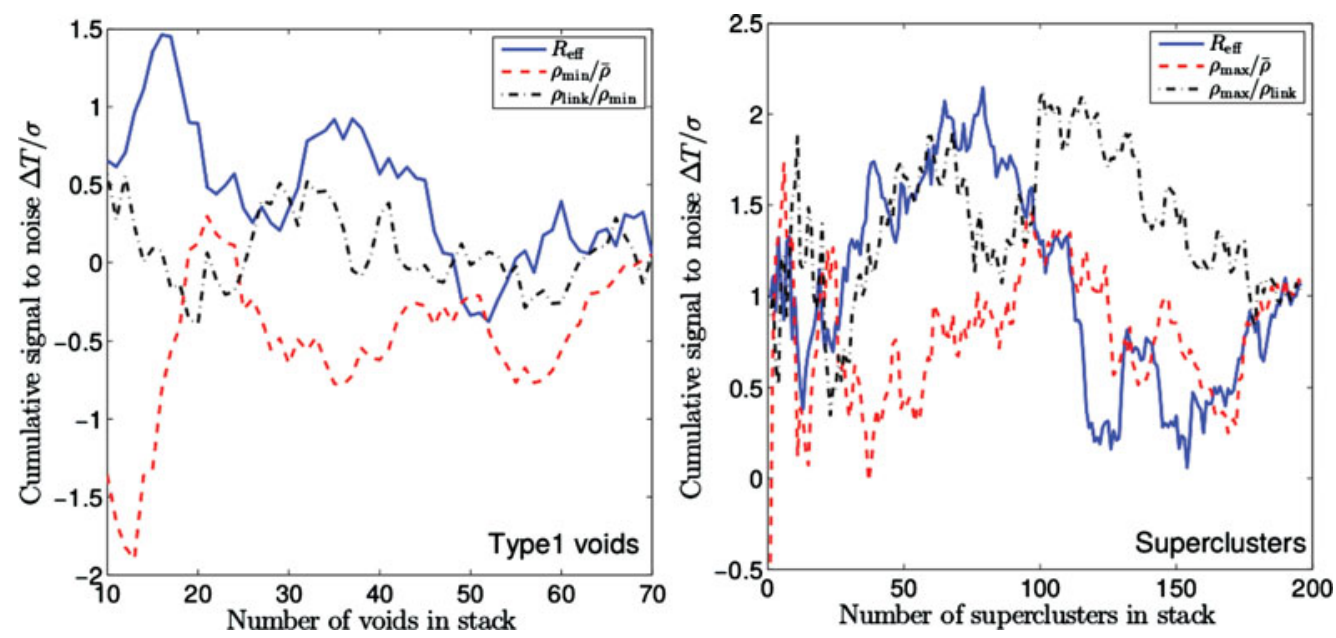

Figure 2. Cumulative S/N values for structures from NH14 and Planck data as the number of structures included in the stack is increased in order of the structure effective radius (blue), maximum density deviation (red dashed) and density contrast (black dot-dashed). Left: For Type1 voids; right: for superclusters.

the Kazin, et al. (2010) samples, labelled lrgdim and lrgbright. For brevity we discuss only results for Type1 voids and superclusters using JDim (lrgdim) here, but results using all the data are described in detail in Hotchkiss et al. (2014).

For each superstructure we extract a patch of the simulated ISW map in that direction and filter it using a compensated top-hat filter

$$
\Delta T\left(\theta_{R}\right)=\frac{\iint_{0}^{\theta_{R}} T(\theta) d \theta d \phi-\iint_{\theta_{R}}^{\theta_{R}^{*}} T(\theta) d \theta d \phi}{\iint_{0}^{\theta_{R}} d \theta d \phi},
$$

where $\theta$ is the azimuthal angle to the line-of-sight through the centre, $\theta_{R}$ is the filter angle and $\theta_{R}^{*}=\arccos \left(2 \cos \left(\theta_{R}\right)-1\right)$. As the structures in the catalogues have very different sizes, the filter radius for each is chosen in fixed proportion to the effective angular size it subtends on the sky, $\theta_{R}=\alpha \Theta_{\text {eff }}$. We then determine the average value $\overline{\Delta T}$ for all superstructures. This value is found to be maximized for relative rescaling $\alpha \simeq 0.6$ (Hotchkiss et al. (2014)), therefore we fix it to this value henceforth. We experimented with the removal of largest scale modes $(\ell \leqslant 10)$ from the map before filtering, and found that removal slightly decreases the total signal amplitude. For superstructures from the SDSS catalogues of NH14 we follow exactly the same procedure except using the Planck SMICA CMB map instead of the simulated ISW-only maps. Errors are estimated by randomizing the lines of sight with the SDSS window (but keeping the same angular size distribution) and calculating the standard deviation over 1000 realizations.

\section{Results}

Jubilee simulation: Fig. 1 shows stacked and rescaled patches of the ISW maps along directions of Type1 voids and superclusters in the Jubilee JDim sample. Void locations clearly correspond to cold spots and supercluster locations to hot spots, as expected. However, the overall magnitude of the average filtered signal is extremely small, corresponding to $\overline{\Delta T} \simeq 0.15 \mu \mathrm{K}$ for both cases (when $\alpha=0.6$ ). This is much smaller than the expected noise in a similar measurement on the CMB due to primary anisotropies at 
last scattering, indicating that the stacked ISW effect of such structures should not be observable in a $\Lambda \mathrm{CDM}$ cosmology.

At the same time, when the structure catalogues are binned according to physical properties, the simulation results show easily understood trends. In particular $\overline{\Delta T}$ increases with the effective radius $R_{\text {eff }}$ of the structure, with decreasing (increasing) minimum (mamimum) density within the void (supercluster), and (more weakly) with increasing density contrast of the structure (see Hotchkiss et al. (2014) for details). These physical trends should be reproduced for any real ISW-like correlation, even if due to some physics beyond $\Lambda$ CDM, but will not be seen for spurious detections due to noise.

SDSS data: We repeated the same procedure for the 70 Type1 voids and 196 superclusters from the lrgdim catalogues of NH14, obtaining $\overline{\Delta T}=0.14 \pm 2.8 \mu \mathrm{K}$ and $\overline{\Delta T}=2.05 \pm 1.9 \mu \mathrm{K}$ respectively, at the rescaling weight $\alpha=0.6$. That is, the results are consistent with zero within the measurement error, exactly in accord with the $\Lambda \mathrm{CDM}$ expectation. Fig. 2 shows the effect on the cumulative $\mathrm{S} / \mathrm{N}$ as the number of structures in each stack is increased from 1 to the final number by adding structures in order of increasing radius, central density deviation and density contrast. In all cases the $\mathrm{S} / \mathrm{N}$ curves are consistent with random walks due to noise alone, i.e. it is not possible to select any physically motivated subset of the observed structure catalogues which provides a significant $\mathrm{S} / \mathrm{N}$. Even allowing the rescaling weight to vary from the optimal value determined from simulation, we do not find an $\mathrm{S} / \mathrm{N}$ value exceeding $2.5 \sigma$ at any value of $\alpha$.

\section{Conclusions}

Our results based on the Jubilee simulation show that the stacked ISW signal of superstructures within $\Lambda$ CDM is orders of magnitude too small to be observable. This vindicates previous estimates (e.g. Nadathur et al. (2012),Flender et al. (2013)) which claimed a discrepancy between the $\Lambda \mathrm{CDM}$ expectation and the G08 detection. Any purported detection of the stacked ISW signal of voids and superclusters, if truly cosmological, must therefore be in contradiction with the $\Lambda$ CDM model.

However, when attempting to reproduce the G08 measurement using an independent catalogue of structures found from SDSS data spanning a lower redshift range than that used by those authors, we fail to detect any significant ISW signal. This is entirely consistent with theoretical expectation. We conclude that whatever the effect seen by G08 was, it does not exist in these independent galaxy samples. Our null result does not definitively exclude the possibility that the original detection was due to some hypothetical new physical effect manifest only at redshifts $\gtrsim 0.5$; however in our opinion it calls into question its likely physical significance.

Finally, although lack of space precludes a full discussion here, in Hotchkiss et al. (2014) we have also considered the significance of the tentative detection reported by Cai et al. (2013). Given the low significance of the reported detection this is completely consistent with our null result. In addition, we find that it cannot be seen as a confirmation of G08 due to significant differences in methodology. Future observations are required to clear up this issue once and for all, but at present the status of stacked ISW detection claims must remain in doubt.

\section{References}

Cai, Y.-C., Cole, S., Jenkins, A., \& Frenk, C. S., 2010, MNRAS, 407, 201

Cai, Y.-C., Neyrinck, M. C., Szapudi, I., Cole, S., \& Frenk, C. S., 2013, ApJ, 786, 110

Dunkley, J., et al., 2009, ApJS, 180, 306 
Flender, S., Hotchkiss, S., \& Nadathur, S., 2013, JCAP, 1302, 013

Granett, B. R., Neyrinck, M. C., \& Szapudi, I., 2008, ApJ, 683, L99

Granett, B. R., Neyrinck, M. C., \& Szapudi, I., 2009, ApJ, 701, 414

Hernández-Monteagudo, C. \& Smith, R. E., 2013, MNRAS, 435, 1094

Hotchkiss, S. et al., 2014, MNRAS in press, arXiv:1405.3552

Hunt, P. \& Sarkar, S., 2010, MNRAS, 401, 547

Ilić, S., Langer, M., \& Douspis, M., 2013, A\& A, 556, A51

Kazin, E. A. et al., 2010, ApJ, 710, 1444

Nadathur, S. \& Hotchkiss, S., 2014, MNRAS, 440, 1248

Nadathur, S., Hotchkiss, S., \& Sarkar, S., 2012, JCAP, 1206, 042

Neyrinck, M. C., 2008, MNRAS, 386, 2101

Planck Collaboration et al., 2013b, ArXiv e-prints, 1303.5079

Planck Collaboration et al., 2013a, ArXiv e-prints, 1303.5062

Watson, W. A. et al., 2014, MNRAS, 438, 412

Zheng, Z., Zehavi, I., Eisenstein, D. J., Weinberg, D. H., \& Jing Y., 2009, ApJ, 707, 554 